\title{
Managing Asthma on the College Campus: Findings of a Texas Pilot Study
}

\author{
Kevin P Collins MSc RRT RPFT AE-C, Debra N Weiss-Randall EdD CHES, and \\ Nicholas R Henry MSc RRT-ACCS RRT-NPS AE-C
}

\begin{abstract}
BACKGROUND: Students attending institutions of higher education have the option of utilizing their student health center (SHC) for asthma management. However, a review of the available literature revealed no previous research as to how SHCs on college campuses in Texas manage students with asthma. This led to the following research questions; how are SHCs in Texas managing asthma for college students, and what are the SHC directors' attitudes and perceptions of the impact of asthma on their college campuses? METHODS: This descriptive pilot study randomly selected 20 colleges in Texas; 17 institutions agreed to participate. The inclusion criteria consisted of 4-y colleges accredited by the Southern Association of Colleges and Schools with an undergraduate student population of greater than 1,000 full-time students, excluding online colleges. Once identified, the director of each institution's SHC was contacted by telephone and invited to complete the Managing Asthma on College Campuses Survey instrument via telephone interview $(n=14)$ or e-mail $(n=3)$. Descriptive statistics were used to analyze quantitative data and common themes were noted for the qualitative data. RESULTS: Quantitative data obtained through the survey revealed $23.5 \%$ of SHCs provide individualized asthma action plans to students and $35 \%$ of SHCs stated they have an emergency action plan for asthma exacerbations. Respondents noted 2 major barriers for effective asthma management on campus: lack of understanding of chronic disease management and underuse of the SHC due to a lack of awareness of its existence. Perceived barriers to visiting the SHC included access issues, money, and students self-medicating with short-acting $\beta 2$ agonist inhalers and/or over-the-counter medications. CONCLUSIONS: The majority of directors were satisfied with asthma services provided to students; however, they felt more face-toface asthma education was needed. Key words: asthma management; college students; student health center; asthma education; expert panel report 3; managing asthma on college campuses survey. [Respir Care 2015;60(8):1085-1090. (C) 2015 Daedalus Enterprises]
\end{abstract}

\section{Introduction}

Asthma is a chronic disease of hyper-reactive airways that is characterized by reversible bronchial smooth muscle constriction, bronchial wall inflammation, and hypersecretion of mucous, resulting in air flow obstruction. ${ }^{1}$ In the United States, the number of people diagnosed with asthma grew by 4.3 million from 2001 to 2009; an estimated 25.7 million persons had asthma in $2010 .^{2}$ Asthma

Mr Collins and Mr Henry are affiliated with the Department of Respiratory Care, Texas State University, San Marcos, Texas. Dr Weiss-Randall is affiliated with the Department of Exercise Science and Health Promotion, Florida Atlantic University, Boca Raton, Florida.

Mr Collins presented a poster and oral presentation of this research at the Annual Conference of the Association of Asthma Educators, held August 1-3, 2014, in San Antonio, Texas. has been shown to have a financial impact on individuals due to higher rates of absenteeism from work and school and decreased productivity of individuals as a result of increased asthma symptoms. ${ }^{3}$ The American College Health Association states that $8.8 \%$ of college students reported ${ }^{4}$ having asthma in 2012; previous studies have shown that college students' quality of life is diminished by asthma symptoms, which affects their self-esteem and academic performance. ${ }^{5,6}$ College students with asthma have been

\footnotetext{
The authors have disclosed no conflicts of interest.

Correspondence: Kevin P Collins, MSc RRT RPFT AE-C, 601 University Drive, Department of Respiratory Care, Texas State University, San Marcos, Texas 78666. E-mail: kc35@txstate.edu.
}

DOI: $10.4187 /$ respcare. 03877 


\section{Managing Asthma on College Campus}

shown to have greater anxiety and psychological distress, and lower health-related quality of life and social functioning, compared with college students without asthma. ${ }^{5,6}$ Misperceptions persist about asthma, making its management more challenging. A study performed by Mancuso et $\mathrm{al}^{7}$ found that $37 \%$ of adults with asthma believed that their asthma would be cured after treatment, and this perception was associated with poorer outcomes.

\section{See the Related Editorial on Page 1211}

College students with asthma have misperceptions of their asthma severity when compared with an objective asthma severity scoring survey. ${ }^{8}$ Furthermore, $40 \%$ of college students do not seek medical assistance even when the student recognizes severe asthma symptoms are present. ${ }^{6}$ The college students' misperceptions of asthma severity and lack of asthma management skills put them at risk for increased hospitalization. ${ }^{9}$ Teenagers may believe their asthma to be an episodic disease, which may lead to noncompliance with their daily medication regimen. ${ }^{10}$ Poor asthma management skills among adolescents could be due to the need for continued assistance from the adolescents' parents and to the nature of asthma's highly visible treatment, which could potentially stigmatize adolescents and isolate them from their peers. ${ }^{10}$

Students attending college may have multiple healthcare resources available to treat and/or manage their asthma, including: the student health center (SHC) on their college campus, their hometown primary care physician, an urgent care center, an asthma specialist, or the local hospital emergency department. However, according to previous literature, poor asthma control and undiagnosed asthma are present among college students with asthma. ${ }^{8} \mathrm{~A}$ review of the literature revealed no previous research as to how SHCs on college campuses in Texas manage students with asthma. This led to the following research questions; how are SHCs in Texas managing asthma for college students, and what are the SHC directors' attitudes and perceptions of the impact of asthma on their student population?

\section{Methods}

This descriptive pilot study surveyed SHC directors at 17 4-y regionally accredited colleges and universities in Texas. To obtain a representative sample of 4 -y accredited colleges and universities in the state of Texas, the investigators utilized the Southern Association of Colleges and Schools online membership list (www.sacscoc.org, Accessed October 12, 2013). The sampling framework consisted of 70 institutions with a full-time enrollment of at least 1,000 undergraduate students, excluding online colleges. Every institution in the sample was assigned a unique

\section{QUICK LOOK}

\section{Current knowledge}

College students with asthma have misperceptions of their asthma severity, and lack of asthma management skills puts them at risk for increased hospitalization. Students have multiple healthcare resources available to treat and/or manage their asthma, including: the student health center (SHC), primary care physician, urgent care center, an asthma specialist, or the local emergency department. Poor asthma control and undiagnosed asthma are present among college students with asthma.

\section{What this paper contributes to our knowledge}

This pilot study reinforces the need for effective asthma management strategies to be implemented at SHCs on college campuses in Texas. Areas identified for further improvement include the education of asthmatic students in chronic disease management, increased faceto-face education, use of individualized asthma action plans, and development of emergency action plans for asthma exacerbations on campus. Marketing and promotion of asthma services available at the SHC is needed to increase student awareness of the treatment and management services offered.

number on the Southern Association of Colleges and Schools list of schools meeting the inclusion criteria. Institutions were selected for participation using a table of random numbers; 20 institutions were selected for this study. The institutions were stratified across 3 dimensions: size, affiliation, and location (Table 1). The size of each institution was defined as follows: small (1,000-1,999 full-time undergraduate student enrollment), medium (2,000-4,999 full-time undergraduate student enrollment), and large (5,000 or more full-time undergraduate student enrollment). The affiliation of the institutions was defined as private or public. The location of the institutions was defined as urban or nonurban. Urban was defined as a greater metropolitan area, including suburbs; nonurban was defined as small towns and rural areas. Based on the information in institutional review board exemption request EXP2013S6911, submitted on July 9, 2013, this research project was exempt from full or expedited review by the Texas State Institutional Review Board. Voluntary consent was obtained from each participant before conducting the survey.

The SHC director of each institution was invited to participate in the study via a telephone interview. Interviews were then conducted using the Managing Asthma on College Campuses Survey (MACCS) (Figure 1). ${ }^{11}$ During the data collection, several SHC directors expressed their willingness to participate in the Texas pilot study but 
Table 1. Profile of the Studied Institutions

\begin{tabular}{lr}
\hline \hline Dimension & $n$ \\
\hline Size & \\
Small & 2 \\
Medium & 1 \\
Large & 14 \\
Affiliation & \\
Public & 11 \\
Private & 6 \\
Location & \\
Urban & 13 \\
Nonurban & 4 \\
\hline
\end{tabular}

noted that they did not have time during the business day to complete a telephone survey. Due to this, the SHC directors were provided the option to complete the interview via email. The MACCS is a 15-item survey questionnaire with closed and open-ended questions. The MACCS instrument comprises 5 domains: (1) school profile; (2) health center staffing patterns; (3) allergy capability; (4) asthma action plans, policies, and information; (5) asthma perceptions and attitudes. The MACCS instrument was used to collect both quantitative and qualitative data in this pilot study. The first 4 domains used quantitative methods; the fifth domain used qualitative methods. The quantitative data analysis assessed the nature and scope of asthma management on 4-y college campuses in the state of Texas, whereas the qualitative data analysis described respondents' perceptions of the impact of asthma on campus and suggestions for improving campus asthma management in the future.

Descriptive statistics were used to analyze quantitative data, and common themes were noted for the qualitative data. The collection of data through interviews or e-mail occurred November 2013 through January 2014 during regular business hours, namely, Monday through Friday between 9 AM and 5 PM, Central Standard Time. Attempts to contact each SHC director were made on different days of the week and at different times of the day; all telephone calls were logged. Each SHC director was called a maximum of 5 times; if the director was reached after fewer attempts, no more calls were made. The telephone interviews were audio recorded, with consent of each participant, and were later transcribed into a Word document for qualitative analysis.

\section{Results}

\section{Quantitative Results}

School Profiles. The response rate of this study was $85 \%$, with 17 SHC directors consenting to participate in the study; 3 SHC directors could not be contacted after 5 attempts. Three SHC directors requested that the survey be sent electronically via email. Questions 1-4 of the MACCS instru- ment comprised the school profile (Table 1). The 17 institutions selected for the sample had a mean of 14,710 full-time students.

Staffing Patterns. Question 5 of the MACCS instrument requested information regarding the staffing for each SHC. The SHC directors had varying backgrounds: 6 medical doctors (MDs), 3 nurse practitioners (NPs), 6 registered nurses (RNs), and 2 PhDs. Fifteen (88.2\%) of the SHC directors were female. Staffing of the SHCs varied considerably. Nine schools $(52.9 \%)$ had full-time medical doctors on staff, and one had a DO in addition to an MD. Six SHCs $(35.3 \%)$ had part-time physicians on staff. Two SHCs $(11.8 \%)$ did not have a physician on staff.

Large institutions were more likely than small or medium institutions to have a physician employed at the SHC. All 14 large institutions in the study had physicians on their SHC staff; 7 of these had full-time MDs, and 7 had part-time MDs. The medium-sized institution SHC and one small institution SHC did not have a physician on staff, whereas the other small institution SHC had a physician on staff $4 \mathrm{~h} / \mathrm{wk}$. All of the public institutions had a physician on staff, and 4 of the private institutions had a physician on staff.

Asthma-Related Visits. When asked, "How many asthma-related visits did the SHC have last year?" only 5 of the 17 SHCs directors responded; the range of responses was from 83 to 648 visits per year. Thus, $29.4 \%$ of the surveyed institutions were able to provide data regarding the number of asthma-related visits during the previous year.

Allergy Capability. Question 6 explores the capability of the SHC to treat allergies. All but one of the health centers $(94.1 \%)$ reported the capability of treating environmental allergies with antihistamines or intranasal steroid sprays. Twelve of the $17 \mathrm{SHCs}(70.6 \%)$ reported having the capability of giving allergy shots.

Asthma Action Plans and Protocols. Four of the 17 SHCs (23.5\%) had adopted an individualized asthma action plan for students to use in the ongoing treatment of their asthma; 35\% of the SHCs stated they have an emergency action plan for asthma exacerbations. Seven of the 17 directors (41\%) revealed on the survey that their SHC had a protocol for handling on-campus asthma attacks. However, the protocols varied among the SHCs. Examples of SHC protocols included: a staff person calling 911, clinical staff administering an aerosolized medication treatment (eg, $2.5 \mathrm{mg}$ of albuterol via nebulizer), and campuspolice transporting the student experiencing an asthma exacerbation from their location on campus and bringing them to the SHC for treatment.

Educational Information. All of the SHCs provided educational materials on asthma. However, the source of the 
1. College/University Information

College/University Name,

Respondent's name, gender, discipline, title and years in position

2. Is the College/University Public or Private?

3. Is the College/Univeristy Urban or Non-Urban?

4. College/University: Number of full time students?

5. Please provide the following information about the Health Services Staff:

Days/hours per week a physician, nurse, nurse practitioner, or physician assistant is on staff?

Please describe the back-up plan for times when none of the above healthcare professionals are on staff (or, if no health center, describe emergency medical procedures).

How many asthma-related visits did the Health Center have last year?

6. A. Does your Health Center have the capability of giving allergy shots?

B. Does your Health Center treat environmental allergies with antihistamines or intranasal steroids or sprays?

7. Has your school adopted an individual asthma action plan for student with asthma to use in their ongoing treatment? If you answered yes, which one do you use?

8. Does the school have an asthma emergency action plan? If you answered yes, please describe it.

9. Do you have a protocol for handling on-campus asthma attacks? If you answered yes, please describe it.

10. Do you provide and educational materials on asthma at the Health Center?

A. Which brochure(s) on asthma do you use?

B. I am satisfied with the brochure(s) on asthma.

Strongly Agree Agree Mixed Feelings Disagree Strongly Disagree

11. Is there any specific information on asthma in the student handbook?

I am satisfied with the asthma information in the student handbook

Strongly Agree Agree Mixed Feelings Disagree Strongly Disagree

12. I am satisfied with the asthma services provided in the Health Center.

Strongly Agree Agree Mixed Feelings Disagree Strongly Disagree

If you answered somewhat satisfied or not satisfied, how could the asthma services at the Health Center be improved?

13. What is your perception of the impact of asthma among students on your campus?

14. Research has shown that $40 \%$ of college students with active asthma symptoms do not seek treatment at their campus's Health Center.

Why do you think this is so? What else do you think students do for asthma treatment?

15. What suggestions do you have for influencing students with asthma to visit your Health Center and manage their asthma on an ongoing basis?

Fig. 1. The Managing Asthma on College Campuses Survey.

educational materials varied among the SHCs. The most frequently sited source (23\%) for educational materials was the American College Health Association's brochure on asthma. Other sources of educational materials included commercially prepared brochures, the SHC's electronic medical record, and websites (eg, Up-to-Date, Centers for Disease Control). Two SHCs created their own educational material, and one director stated she did not know which educational material was being provided to educate students on asthma management.

\section{Qualitative Results}

Asthma Perceptions and Attitudes. Questions 11-14 of the MACCS instrument documented each SHC director's asthma perceptions and attitudes. Twelve of the directors (70.6\%) were satisfied with the asthma services provided by their SHC, 3 (17.6\%) had mixed feelings, and 2 (11.8\%) did not answer the question. Those who had mixed feelings noted that they were trying to more fully develop treatment plans that followed the 2007 Expert Panel Report 3 guidelines published by the National Heart, Lung and Blood Institute, ${ }^{12}$ including the use of written asthma action plans. Another recommendation for improving asthma services was to create more consistency among providers (standardization of care), which is facilitated by the use of written asthma and emergency plans.

Question 13 asked the SHC directors their perception of the impact of asthma among students on campus. One SHC director at a public university with almost 40,000 students noted that asthma on campus "is a major health problem, underdiagnosed and undertreated." Eight of the SHC respondents (47.1\%) perceived the impact of asthma to be seasonal; 4 (23.5\%) reported the impact of exercise- 


\section{Managing Asthma on College Campus}

Table 2. Quotes Illustrating Barriers to Seeking Treatment at 4-y College Student Health Centers in Texas

\begin{tabular}{|c|c|}
\hline Barrier & Quote \\
\hline $\begin{array}{l}\text { Students' lack of understanding of } \\
\text { chronic disease management }\end{array}$ & $\begin{array}{l}\text { "Freshmen are used to depending on their parents for the answers to their healthcare, } \\
\text { so they haven't learned to be independent health consumers." } \\
\text { "They just live through it and don't seek treatment anywhere." } \\
\text { "Horrifyingly sometimes they go buy Primatene Mist or other OTC meds that do not } \\
\text { work; use old inhalers, borrow inhalers." }\end{array}$ \\
\hline Money issues/lack of health insurance & $\begin{array}{l}\text { "Here it's because the students generally do not have insurance." } \\
\text { "A lot of them don't have money, so they just want albuterol, just the cheapest one they can get." }\end{array}$ \\
\hline Accessibility & "Convenience, they may have a wait time. It may be due to the center not being open on the weekends." \\
\hline Inaccurate diagnosis & $\begin{array}{l}\text { "They haven't been accurately diagnosed. They believe they had asthma in the past or } \\
\text { that they have recurrent bronchitis which is in fact asthma." }\end{array}$ \\
\hline Lack of awareness of SHC services & "Most don't utilize their campus health services because they are not aware that it is there." \\
\hline Lack of confidence in SHC & "I think it may be due to old misperceptions of university student health centers." \\
\hline $\begin{array}{l}n=17 \\
\text { OTC }=\text { over the counter } \\
\text { SHC }=\text { student health center }\end{array}$ & \\
\hline
\end{tabular}

induced asthma. Climate was also cited as a factor. In southern Texas, the humid, moist environment was described as a trigger for asthma exacerbations even in students who had not experienced asthma symptoms for several years. Likewise, the desert climate of western Texas was described as a rough environment for those with asthma, with one SHC director reporting seeing approximately 500 cases of asthma each month.

Research has shown that $40 \%$ of college students with active asthma symptoms do not seek treatment at their SHC. ${ }^{6}$ Question 14 asked: (1) why do you think this is so? and (2) what else do you think students do for asthma treatment? Table 2 lists the 6 categories of responses from the directors, along with selected quotes illustrating the barriers to seeking treatment for asthma at SHCs.

Finally, question 15 asked whether the respondent had any suggestions for influencing students with asthma to visit the SHC. All of the respondents stated that effective asthma education was the key to better utilization of campus health services, and they felt that more face-to-face education of both students and parents was needed. They also commented on the need for better health insurance coverage, so that students with asthma would refill their prescriptions, which are often costly. Many SHC directors acknowledged they need to make the SHC more visible, so that students know it is there and what services it offers. Some used social media such as Twitter and Facebook for health promotion.

\section{Discussion}

The pilot study results documented the scope and nature of asthma management on college campuses in the state of Texas. It was undertaken to attempt to replicate the results of the 2010 study in New York state, which was the first to develop and use the MACCS instrument. ${ }^{11}$ Results of our study reveal improvements are needed in the following areas (1): more face-to-face education regarding chronic disease management, (2) promotion of the asthma services offered by the SHC, (3) the use of individualized asthma and emergency action asthma plans, and (4) better recording of asthma-related visits to the SHC.

All of the institutions included in the Texas pilot study had a SHC, and all had identified a staff person to coordinate the program; in contrast, in the New York study, $82.1 \%$ of the schools had a health center and coordinator. ${ }^{11}$ The differences are likely due largely to size: the mean undergraduate population was 14,710 in the Texas pilot study and 4,952 in the New York study. ${ }^{11}$ In the New York study, $32.5 \%$ of the institutions were small and $32.5 \%$ were medium-sized; only $35 \%$ were large, compared with $82.4 \%$ in the Texas study. Both studies found that large institutions were more likely than small or medium-sized institutions to have a physician on staff at the SHC. In the New York study, $83 \%$ of the schools provided asthma materials ${ }^{11}$ versus $100 \%$ in the Texas study. The consensus in both studies was that more face-toface asthma education of students and parents was needed to improve understanding about chronic disease management and to inform students of the asthma services offered by the SHC. Lack of awareness of the health center and its services was a problem noted frequently in both studies. In addition, both studies found that the vast majority of SHCs did not keep records on asthma-related visits. A survey of medical directors at 200 4-y United States college student health centers found relatively few have a system in place to identify the asthmatic student when he or she arrives on campus. ${ }^{12}$ This study showed many SHCs have the capacity to provide primary care and management of chronic conditions; however, no guidelines are available to assist the student in the transition from asthma management provided by their pediatrician to adult care offered at the SHC. ${ }^{12}$ 


\section{Managing Asthma on College Campus}

National asthma guidelines recommend the use of individualized asthma action plans for all students with asthma. ${ }^{13}$ However, in both the New York and Texas studies, fewer than one-quarter of the schools utilized individualized written asthma action plans for students. Healthy People 2020, the health blueprint for the United States, follows National Asthma Education and Prevention Program guidelines, which emphasize the importance of written asthma plans. Healthy People 2020 objective RD-7.1 is to increase the proportion of persons with current asthma who receive written asthma management plans from their healthcare provider. (www.healthypeople.gov/2020, Accessed October 17, 2013). In Texas, only 35\% of the schools reported using emergency action plans, whereas the figure was closer to one-quarter for the New York study.

The Texas pilot study succeeded in replicating the New York findings. The majority of respondents in both studies reported that they were satisfied with the services offered. However, the disparity in asthma management on college campuses may be improved by implementation of: (1) more face-to-face education of students about chronic disease management, (2) better promotion of the asthma services offered by the SHC, (3) the use of individualized asthma action plans and emergency action asthma plans (protocols), and (4) better recording of asthma-related visits to the SHC.

\section{Limitations}

This study has limitations due to the cross-sectional design; associations can be made, but no causality can be inferred. Three researchers, as opposed to a single researcher, administered the MACCS instrument, so bias of the researcher may have influenced the results of the qualitative data. Not all of the respondents completed the MACCS instrument via telephone interview; 3 completed it via e-mail, thereby possibly limiting the qualitative data collected from those directors. The sample was small; a study with a larger sample size of 4-y colleges in Texas would provide more data and enhance the generalizability of the findings. A future longitudinal study is warranted.

\section{Conclusions}

As previously mentioned, the American College Health Association findings in 2012 stated $8.8 \%$ of college students reported having asthma. With the gross number of asthmatic students on a college campus, it is likely some will visit the SHC for treatment and management of their asthma. This pilot study reinforces the need for effective asthma management strategies to be implemented at SHCs on college campuses in Texas. Key findings reveal a potential for further improvement in the education of the asthmatic student in chronic disease management, increased face-to-face education of students, use of individualized asthma action plans, and development of emergency action plans for asthma exacerbations on campus. Marketing and promotion of asthma services available at the SHC are needed to increase student awareness of the treatment and management services offered at the SHC.

\section{REFERENCES}

1. Des Jardins T, Burton GG. Asthma. In: Des Jardins T, Burton GG. Clinical manifestations and assessment of respiratory disease, 6th edition. Maryland Heights, MO: Mosby; 2011:187-188.

2. Akinbami LJ, Moorman JE, Bailey C, Zahran, HS, King M, Johnson CA, Xiang Liu X. Trends in asthma prevalence, health care use, and mortality in the United States, 2001-2010. NCHS data brief 94. Hyattsville, MD: National Center for Health Statistics; 2012.

3. Lugogo NL, Kraft M, Castro M. Epidemiology of asthma. In: Castro M, Kraft M. Clinical asthma. Philadelphia, PA: Mosby; 2008:3-12.

4. American College Health Association. American College Health Association national college health assessment II: undergraduate reference group executive summary spring 2012. Hanover, MD: American College Health Association; 2012.

5. Fedele DA, Mullins LL, Eddington AR, Ryan JL, Junghans AN, Hullmann SE. Health-related quality of life in college students with and without childhood-onset asthma. J Asthma 2009;46(8):835-840.

6. Carpentier MY, Mullins LL, Van Pelt JC. Psychological, academic, and work functioning in college students with childhood-onset asthma. J Asthma 2007;44(2):119-124.

7. Mancuso CA, Rincon M, McCulloch CE, Charlson ME. Self-efficacy, depressive symptoms, and patients' expectations predict outcomes in asthma. Med Care 2001;39(12):1326-1338.

8. Reece SM, Holcroft C, Faul M, Quattrocchi N, Nicolosi R. A look at asthma care in a university setting. Nurse Pract 2002;27(12):35-42.

9. Mullins LL, Chaney JM, Pace TM, Hartman VL. Illness uncertainty, attributional style, and psychological adjustment in older adolescents and young adults with asthma. J Pediatr Psychol 1997;22(6):871-880.

10. Price JF. Issues in adolescent asthma: what are the needs? Thorax 1996;51(Suppl 1):S13-S17.

11. Weiss, DN. Managing asthma on college campuses: what is being done and what is not being done? Teachers College, Columbia University, ProQuest, UMI Dissertations Publishing, 2010. 3424921.

12. Lemly DC, Lawlor K, Scherer EA, Kelemen S, Weitzman ER. College health service capacity to support youth with chronic medical conditions. Pediatrics 2014;134(5):885-891.

13. National Asthma Education and Prevention Program, Third Expert Panel on the Diagnosis and Management of Asthma. Bethesda, MD: National Heart, Lung, and Blood Institute;2007

This article is approved for Continuing Respiratory Care Education credit. For information and to obtain your CRCE

(free to AARC members) visit www.rcjournal.com

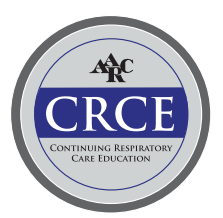

